Agriculture

Publication 1890/F
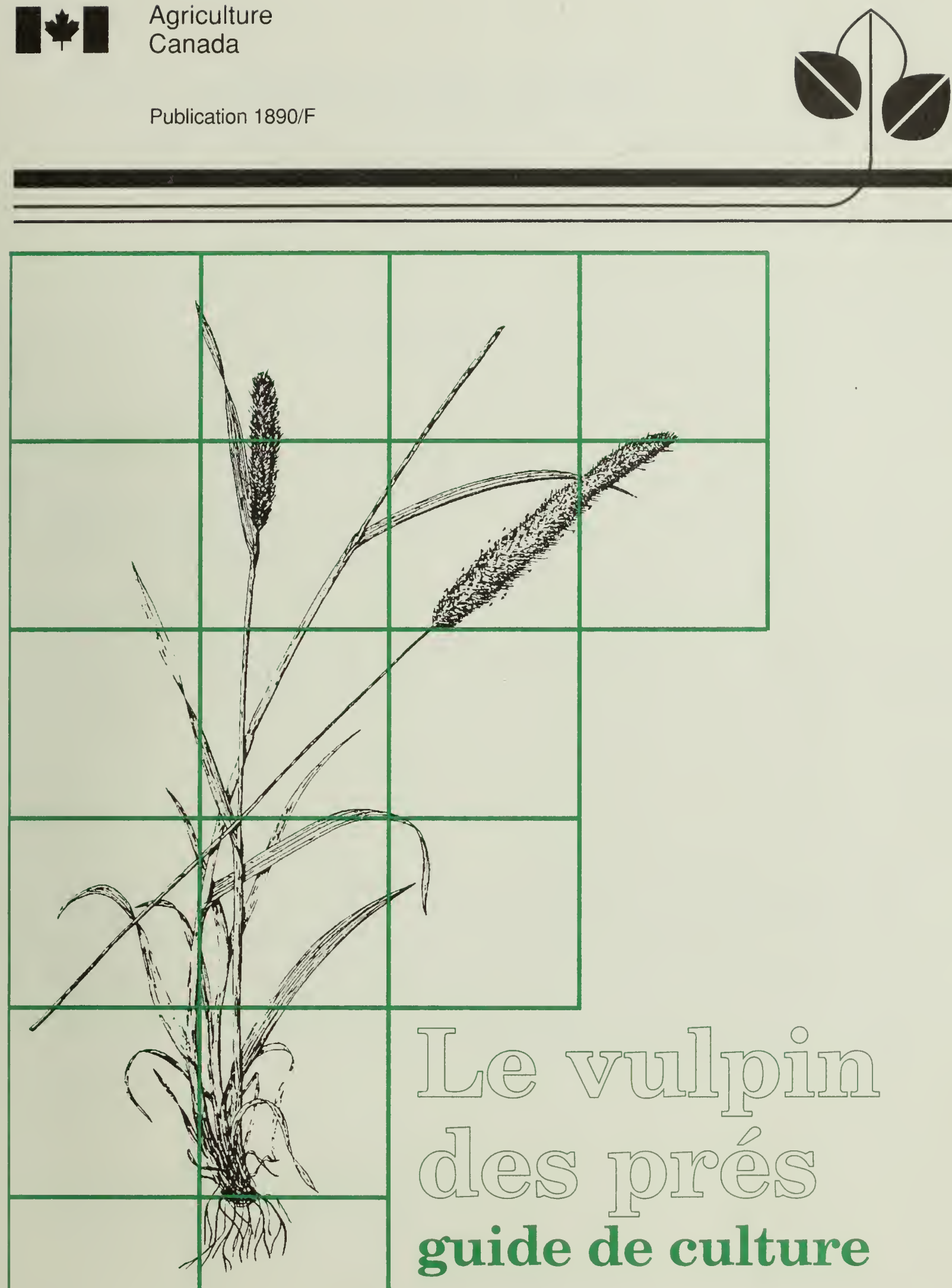

Canadä 



\section{Le vulpin des prés guide de culture}

\section{Patricia Kline}

Ferme expérimentale

Prince George (Colombie-Britannique)

\section{Klaas Broersma}

Station de recherches

Kamloops (Colombie-Britannique)

\section{Scott B.M. Wright}

Station de recherches

Melfort (Saskatchewan)

\section{Lyle M. Rode}

Station de recherches

Lethbridge (Alberta)

Les pesticides recommandés dans la présente publication ne le sont qu'à titre indicatif. L'application doit toujours être conforme aux instructions de l'étiquette du pesticide, comme le prescrit la Loi sur les produits antiparasitaires. Toujours lire l'étiquette. Le pesticide doit également être recommandé par les autorités provinciales. Comme les recommandations d'utilisation peuvent varier d'une province à l'autre, consulter le conseiller agricole de la province pour obtenir des conseils précis.

\section{Agriculture Canada Publication 1890/F}

On peut obtenir des exemplaires à la

Direction générale des communications

Agriculture Canada, Ottawa (Ont.) K1A 0C7

CMinistre des Approvisionnements et Services Canada 1993

No de cat. A53-1890/1993F ISBN 0-662-97994-X

Impression $1993 \quad$ 2M-03:93

Production du Service aux programmes de recherches

Also available in English under the title

Meadow foxtail: a production guide 
Digitized by the Internet Archive in 2012 with funding from

Agriculture and Agri-Food Canada - Agriculture et Agroalimentaire Canada 


\section{Table des matières}

Questions et réponses au sujet du vulpin des prés 6

\section{Introduction 7}

Description 9

Adaptation 9

Encadré 1 Au Canada, le vulpin des prés a sa place... 10

Cultivars 10

\section{Régie 12}

Établissement 12

Lutte contre les mauvaises herbes 12

Encadré 2 Établissement facilité par l'emploi de semences enrobées 13

Mélanges avec des légumineuses et d'autres plantes fourragères Encadré 3 De l'azote, il en faut, mais pas trop non plus 14 Fertilisation et rendement en matière sèche 15

\section{Utilisation 16}

Qualité 16

Pâturage et production de foin ainsi que d'ensilage

Encadré 4 Une question de goût! 20

\section{Production semencière 21}

Récolte 21

Manutention des semences 21

Après le moissonnage-battage 22

\section{Autres utilisations 22}

Recommandations pour l'emploi du vulpin des prés 23

Remerciements 23

Pour en savoir plus 24

Table de conversion vers le système métrique $\quad 25$ 


\section{Questions et réponses au sujet du vulpin des prés}

Où peut-on cultiver le vulpin des prés?

On peut cultiver le vulpin des prés dans des endroits où il y a des inondations causées soit par un mauvais drainage interne soit par une nappe phréatique élevée sur des sols organiques (tourbe) ou d'une texture lourde (argile). Il convient bien à des climats frais et tempérés (voir «Introduction, Adaptation»).

\section{Faut-il utiliser des grains enrobés?}

Le grain du vulpin des prés est léger et duveteux ce qui le rend difficile à semer avec de l'équipement ordinaire. Les grains enrobés peuvent être semés d'une façon plus égale avec de l'équipement ordinaire et leur établissement est amélioré. (voir « Régie, Établissement»).

La qualité et le rendement du vulpin des prés soutiennent-ils la comparaison avec les autres graminées?

Lors d'une évaluation de sept espèces de graminées à sept endroits choisis au centre de la Colombie-Britannique, le vulpin des prés a eu un rendement en matière sèche de $68 \%$ du phalaris roseau et $72 \%$ de la fléole des prés. Cependant, la matière sèche digestible in vivo, la concentration de protéines brutes et le contenu minéral de phosphore, de potassium, de cuivre et de zinc est supérieur aux autres graminées évaluées (voir "Régie, Fertilisation et rendements en matière sèche» et "Utilisation»).

Le vulpin des prés peut-il servir de foin, d'ensilage ou de fourrage sur pied?

Même si le vulpin convient le mieux au pâturage, il peut également servir de foin et d'ensilage. Grâce à sa croissance rapide, il peut être pâturé avant les autres herbes. Si l'on s'en sert comme foin et ensilage, il faut le couper tôt et plus souvent que la plupart des autres graminées, parce qu'il arrive à maturité beaucoup plus tôt (voir les paragraphes consacrés au pâturage et à la production de foin ainsi que d'ensilage).

\section{Quelle est l'appétibilité du vulpin des prés pour les bovins?}

Sur le plan chimique, le vulpin semble une excellente plante fourragère, riche en éléments nutritifs. Toutefois, les essais ont montré que, lorsqu'ils ont le choix, les bovins et les ovins préferrent souvent d'autres graminées. Cette préférence proviendrait surtout du fait que le vulpin arrive à maturité beaucoup plus tôt que les autres graminées et que ce stade est largement dépassé lorsqu'il est récolté (voir les paragraphes consacrés au pâturage et à la production de foin ainsi que d'ensilage). 


\section{Introduction}

Le vulpin des prés (Alopecurus pratensis L.) est une graminée vivace (fig.1) qui convient bien au pâturage au début du printemps dans les basses terres très humides. L'Alopecurus, vient du grec alopex, qui signifie renard et oura, qui signifie queue; vulpin signifie de renard, à cause de la forme de l'inflorescence. D'autres graminées portent des noms qui viennent de la forme de l'inflorescence : l'orge queue d'écureuil (Hordeum jubatum L.), dont les longues barbes (fig. 2) peuvent causer des blessures au bétail; la sétaire verte (Setaria viridis [L.] Beauv.) (fig. 2) ou glauque (Setaria glauca [L.] Beauv.), mauvaises herbes qui infestent les cultures annuelles, dont le nom vient de seta, soies. L'épi de ces graminées ressemble même davantage à la queue d'un renard que l'épi du vulpin. L'épi du vulpin est facilement confondu avec celui de la fléole des prés (Phleum pratense L.) (fig. 2). Au toucher cependant, il est plus velouté que l'épi aux poils raides de la fléole. Le vulpin n'est apparenté à aucune des graminées précitées.

Le vulpin des prés (fig. 2) ressemble à son cousin Alopecurus arundinacea Poir., par sa croissance et son adaptation. Même si beaucoup des pratiques culturales et des emplois cités s'appliquent à ces deux graminées, les renseignements que nous donnons ici proviennent uniquement d'essais effectués avec le vulpin des prés. En culture intensive, celui-ci peut être une herbe très utile qui se prête particulièrement bien aux terrains humides et au pâturage au début du printemps.

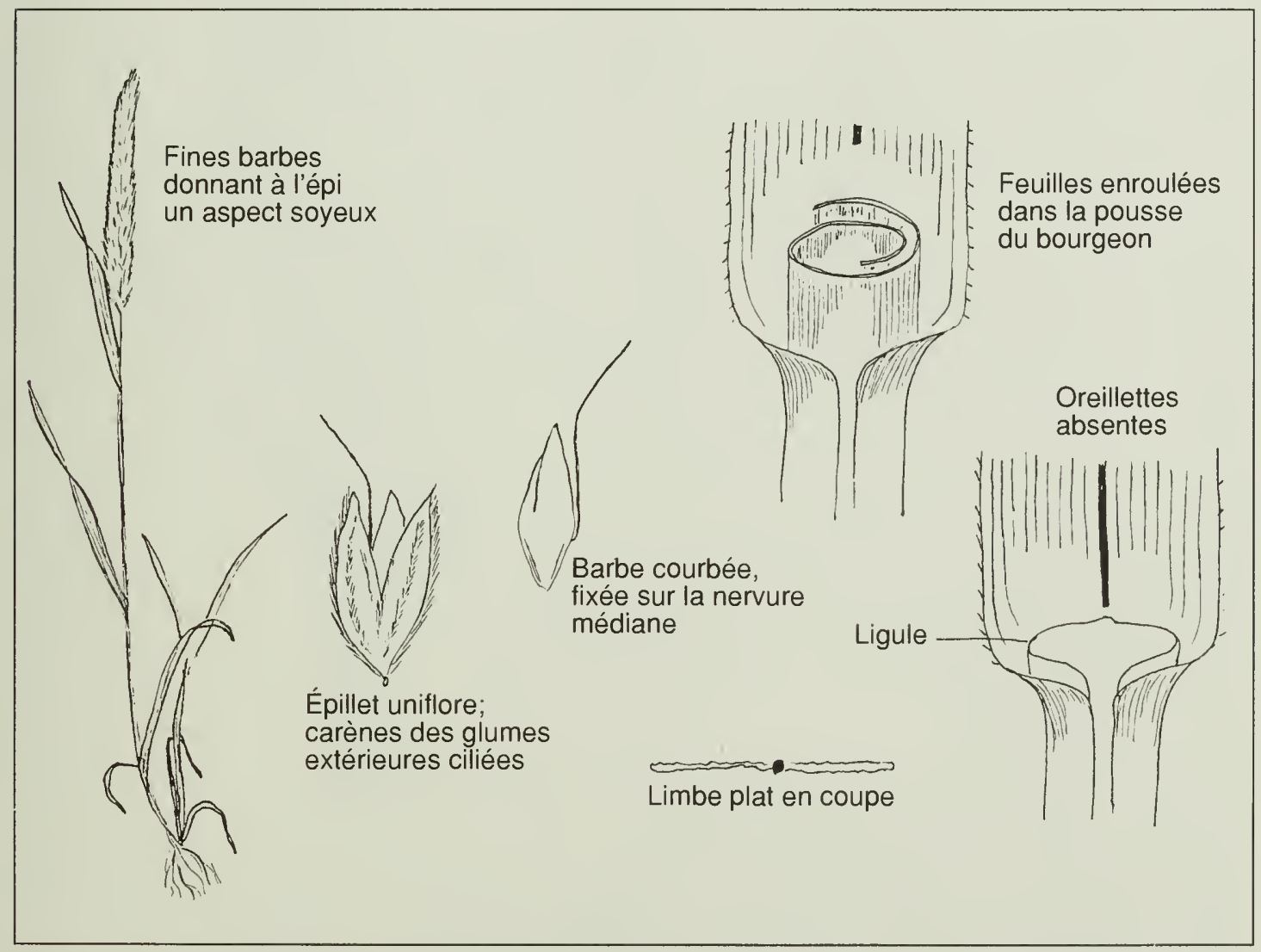

Fig. 1 Caractéristiques du vulpin des prés (dessins réalisés par P. Kline, d'après Herbert B. Hartwig, 1950. Picture aids to grass classification. Ithaca, N.Y.). 

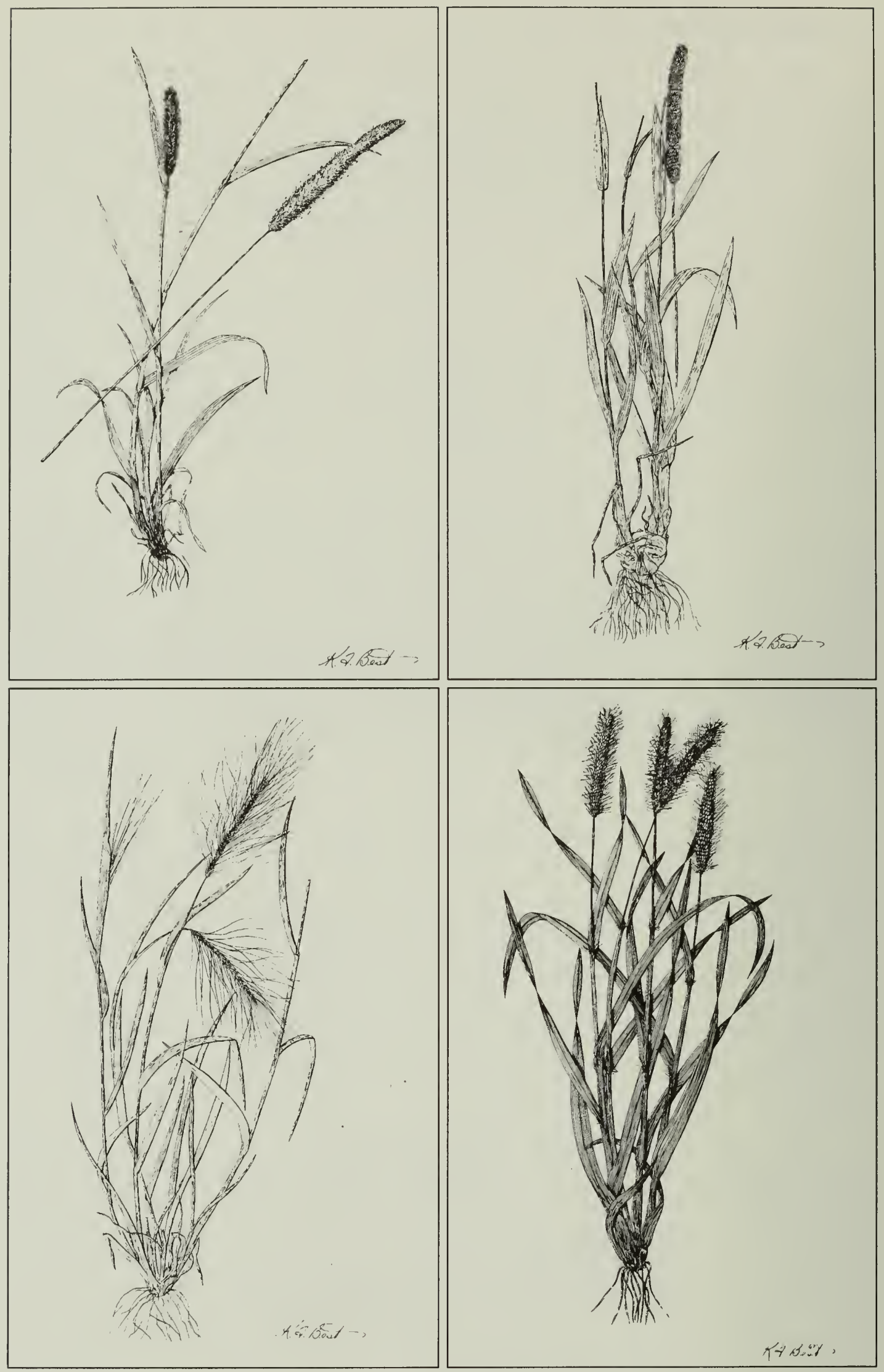

Fig. 2 Vulpin des prés (en haut à gauche), fléole des prés (en haut à droite), orge queue d'écureuil (en bas à gauche), et sétaire glauque (en bas à droite). (D'après Looman, J. 1982. Prairie grasses identified by vegetative characters. Agriculture Canada Publication 1413). 


\section{Description}

Le vulpin des prés est remarquable par sa couleur vert foncé, qui ressort dans les cultures mélangées d'autres plantes fourragères. En hauteur, il atteint 30 à $80 \mathrm{~cm}$. Le pied comporte une forte proportion de feuilles basilaires. Lorsqu'ils lèvent, les nombreux limbes sont enroulés dans le bourgeon et, à maturité, chacun d'eux peut mesurer jusqu'à $1,0 \mathrm{~cm}$ de largeur.

Les inflorescences sont dressées. Les poils fins des glumes et les longues barbes recourbées des graines leur donnent une apparence soyeuse. Les graines sont légères, duveteuses et de couleur pâle.

Les ramifications souterraines et les rhizomes sont courts, non envahissants. Même si le vulpin des prés forme des touffes peu denses, on trouve dans les vieux peuplements des plaques gazonnées de densité moyenne (fig. 2).

\section{Adaptation}

Le vulpin des prés est cultivé depuis environ 250 ans, mais il a été introduit en Amérique du Nord uniquement à la fin du siècle dernier, par un immigrant d'Europe orientale. Aujourd'hui, on peut l'observer, qui croît librement dans beaucoup de terrains bas et humides, habitat auquel il est le mieux adapté.

En Amérique du Nord, il est particulièrement adapté aux climats tempérés et frais du Canada et du nord des États-Unis (voir encadré 1). Il prospère dans les zones humides et croît naturellement dans les terrains marécageux et près des étangs et des cours d'eau. Capable de survivre aux submersions, cette graminée est bien adaptée aux pelouses tourbeuses, dont le sol est très humide et riche en matière organique.

À cause de sa tolérance au gel et de sa capacité de croître sous un climat frais, le vulpin des prés est l'une des premières graminées à verdir au printemps (planche 1a). Il est souvent en pleine croissance quand la fonte le débarrasse de son manteau de neige et il reprend vigoureusement vie après un cycle de gel et de dégel ou après avoir été recouvert de glace. Cette caractéristique procure à l'éleveur l'avantage supplémentaire de faire pâturer ses animaux tôt au printemps (planche $1 b$ ).

Intolérant à la sécheresse, le vulpin des prés convient mal aux régions exposées à de longues disettes d'eau. Si le sol perd de son humidité, cette graminée entre en une semi-dormance qui se prolonge jusqu'à ce que les conditions s'améliorent. Le vulpin croît le mieux dans les régions où l'humidité est constamment appropriée. Il réagit à l'irrigation, mais il n'a pas été cultivé de façon extensive sous régime irrigué.

Durant les années humides, la croissance printanière et le rendement annuel peuvent être intenses, la première ne souffrant aucunement de la submersion. Le vulpin des prés peut survivre sans dommage à un séjour dans l'eau froide à une profondeur de 60 à $90 \mathrm{~cm}$ pour une période qui peut atteindre 30 jours. Il prospère s'il subit un certain degré de submersion, comme le montre l'accélération ultérieure 
Encadré 1 Au Canada, le vulpin des prés a sa place ...

- en Colombie-Britannique

Le vulpin des prés est bien adapté aux sols acides, mal égouttés et frais du centre de l'intérieur de la Colombie-Britannique. Pendant plus de 25 ans, on a étudié sa croissance et son utilisation à la Ferme expérimentale de Prince George, dans des sols argileux lourds. Lorsqu'il bénéficie d'une fumure azotée (printanière et après chaque fauche, jusqu'à concurrence de trois fauches), le vulpin des prés peut produire jusqu'à $6885 \mathrm{~kg}$ de matière sèche par hectare et par année.

- dans le nord de l'Alberta

La Pembina Forage Association a signalé un gain de poids satisfaisant chez les bouvillons mis à paître au vulpin des prés dans des études du pâturage en rotation effectuées dans des pelouses humides. Á Beaverlodge (Alberta), des parcelles expérimentales ont eu un rendement annuel en matière sèche de plus de $13000 \mathrm{~kg} / \mathrm{ha}$, après trois fauches.

- et en Ontario

Des expériences effectuées par l'Ontario Forage Council ont montré que le vulpin pouvait donner un rendement supérieur à celui de la fléole, quand sa régie était effectuée de façon avisée. Dans la vallée de la rivière des Outaouais, on a atteint un rendement annuel de plus de $10000 \mathrm{~kg} / \mathrm{ha}$. À New Liskeard et à Kapuskasing, on a obtenu expérimentalement un rendement annuel d'environ $6000 \mathrm{~kg}$ de matière sèche à l'hectare.

de la croissance, ce qui lui procure un avantage sur les autres espèces. Dans les terrains humides, les carex et les joncs indigènes se laisseront dominer par lui.

Le vulpin des prés tolère les sols acides et alcalins. Il croît bien lorsque le $\mathrm{pH}$ se situe entre 5,1 et 8,4 . Sa tolérance aux sols alcalins dépend directement de l'humidité disponible du sol. La salinité du sol nuit à sa croissance et à son développement.

\section{Cultivars}

La plupart des semences de vulpin des prés utilisées au Canada appartiennent à la catégorie ordinaire Canada no 1 . Deux cultivars seulement, le Dan et le Mountain, sont actuellement homologués au pays.

Homologué en 1987, le Dan est d'origine polonaise.

Homologué en 1989, le Mountain a été créé par O. Pick and Sons Seeds. Il 

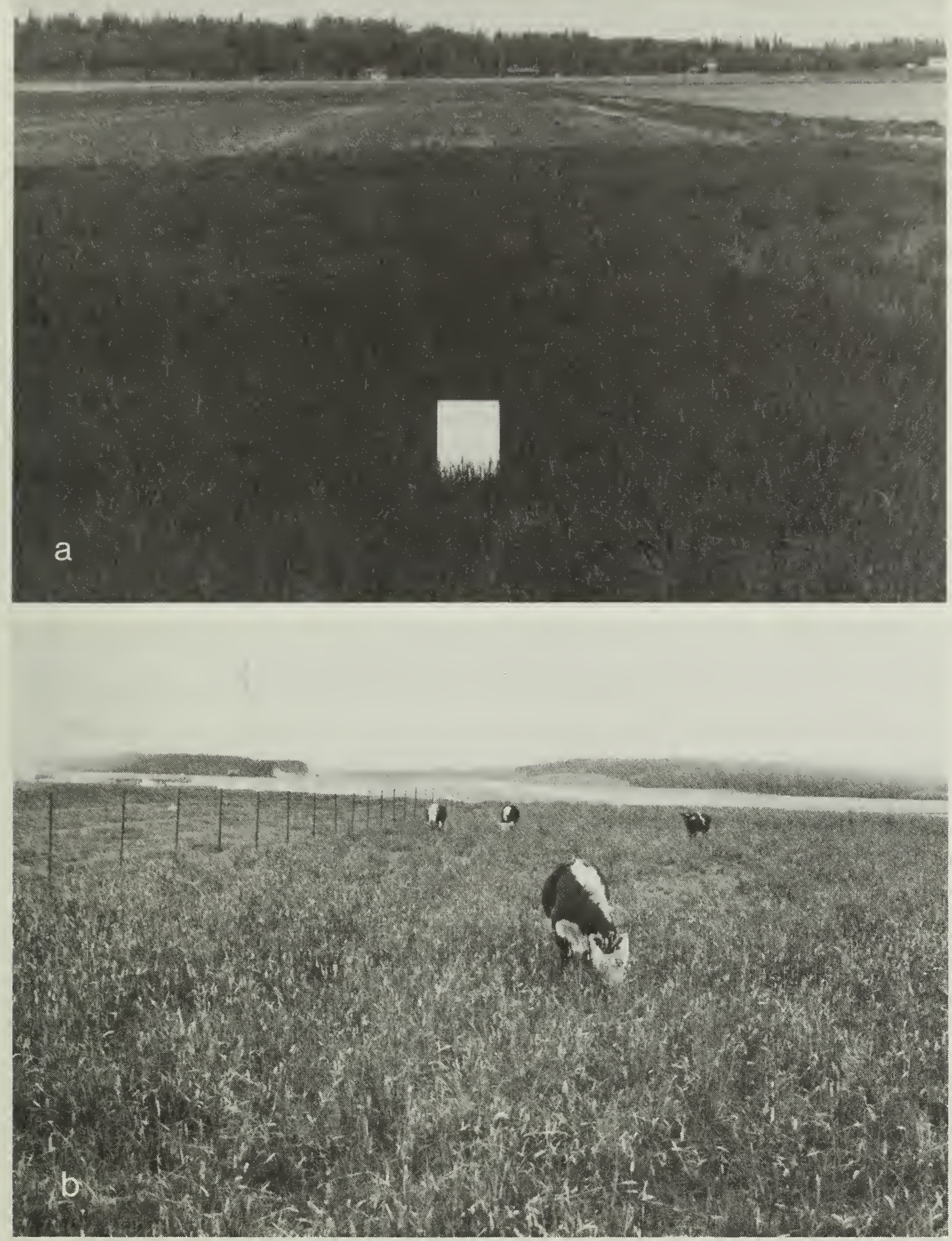

\section{Planche 1}

$a$ Le vulpin des prés (au premier plan) est la première graminée à verdir au printemps.

$b$ Le vulpin des prés peut être brouté plus tôt que les autres graminées. 
a été sélectionné pour sa croissance printanière précoce, qui se poursuit en arrière-saison, pour son rendement semencier et parce qu'il est exempt de maladies foliaires.

Chez les deux cultivars, la vitesse d'épiaison, le rendement, la rusticité hivernale et la qualité chimique sont semblables.

\section{Régie}

\section{Établissement}

Les terrains idéals pour cette graminée comprennent les sols tourbeux et marécageux, les terrains vaseux, les plaines d'inondation (lits majeurs) des cours d'eau, où la nappe phréatique est élevée, ainsi que d'autres types de terrains mouillés. Dans la plupart des endroits, l'ensemencement printanier convient le mieux. Toutefois, comme le vulpin est le mieux adapté aux terrains humides, il peut être impossible d'amener sur place le matériel au printemps. On peut alors retarder l'ensemencement jusqu'à ce que le sol soit praticable, tout en étant encore humide et jusqu'à ce qu'il reste 6 ou 8 semaines de croissance végétative avant les gelées rigoureuses. L'ensemencement d'automne ou du début de l'hiver est couronné de succès s'il a lieu suffisamment tard pour que la germination soit retardée jusqu'au printemps.

Ensemencer à la profondeur de 0,5 à $1 \mathrm{~cm}$, à espacement de $15 \mathrm{~cm}$ entre les rangs; le lit de germination doit être ferme et humide. Il importe de semer à faible profondeur et de tasser le sol ensuite, notamment dans les sols tourbeux et marécageux. L'espacement préconisé empêche l'infestation par les mauvaises herbes, problème répandu durant l'établissement de la plante. On peut également semer à la volée, puis incorporer les semences au sol par un hersage léger.

Si on utilise des semences nues, y aller à la dose de 9 à $13 \mathrm{~kg} / \mathrm{ha}$, mais augmenter la dose de façon à corriger l'augmentation du poids lorsqu'on utilise des semences enrobées (voir encadré 2). Toujours vérifier le taux de germination de la semence au préalable parce que ce taux diminue rapidement à mesure que la semence vieillit. Corriger en conséquence les doses de semis. La levée se produit au cours des 2 semaines qui suivent lorsque l'humidité et la température du sol sont suffisantes.

\section{Lutte contre les mauvaises herbes}

Lorsque le vulpin des prés est cultivé pour la production de semences, il faut combattre les mauvaises herbes. S'assurer que les semences et les champs sont aussi exempts que possible de mauvaises herbes. Avant l'ensemencement, on peut travailler le sol de la façon convenable ou utiliser un herbicide tel que le glyphosate, afin de combattre les mauvaises herbes telles que le chardon des champs, la linaire et le chiendent. Quand la culture est établie, les méthodes de lutte chimique sont limitées. Les seuls herbicides homologués pour les champs de vulpins des prés 
- Les semences du vulpin des prés, légères, duveteuses, peuvent être difficiles à répartir uniformément à l'aide des semoirs ordinaires. Si elles sont enrobées de carbonate ou de phosphate de calcium et de magnésium, les semences seront plus faciles à mieux répartir. Corriger la dose de semis en tenant compte du fait que le quart seulement du poids des semences enrobées est constitué de la semence.

- Des expériences effectuées à la Ferme expérimentale de Prince George ont montré que l'enrobage a légèrement abaissé le taux de germination du vulpin, qui était de $65 \%$ chez les semences nues et de $55 \%$ seulement chez les semences enrobées. Malgré cette baisse dans les conditions de laboratoire, on n'a observé, en plein champ, aucun écart significatif entre les semences nues et enrobées dans le rendement en matière sèche ni dans la teneur en protéines.

semenciers sont le Basagran et le Lontrel. Les autres herbicides peuvent endommager les épis et ainsi abaisser le rendement semencier.

Pour la production fourragère, on peut compter sur un nombre beaucoup plus grand de produits contre les mauvaises herbes à feuilles larges, mais les recommandations varient localement. Consulter le service de vulgarisation agricole de la province pour connaître celles qui visent la localité.

\section{Mélanges avec des légumineuses et d'autres plantes fourragères}

En peuplement mélangé, le vulpin des prés pousse très vite, de sorte qu'il est prêt à être brouté au moment où les autres plantes fourragères commencent à peine à pousser. Si l'on attend que les autres plantes fourragères le rejoignent, le vulpin aura dépassé le stade optimal de la maturité. Le bétail préférera donc les espèces qui n'ont pas atteint la maturité et il dédaignera le vulpin.

Les terrains humides propices au vulpin des prés se prêtent généralement mal à la culture des légumineuses. On a utilisé jusqu'à un certain degré le lotier corniculé ainsi que le trèfle alsike, plantes qui, plus que les autres légumineuses, peuvent tolérer les milieux humides. Toutefois, ils commencent à croître beaucoup plus tard que le vulpin. Le lotier se laisse complètement dominer par le vulpin. Il est donc difficile à implanter en mélanges de semences. Dans les peuplements mélangés de vulpin des prés et de lotier corniculé, à Prince George, l'alternance des rangs de chaque espèce ou le semis croisé ont permis de réduire la concurrence entre les deux espèces. 


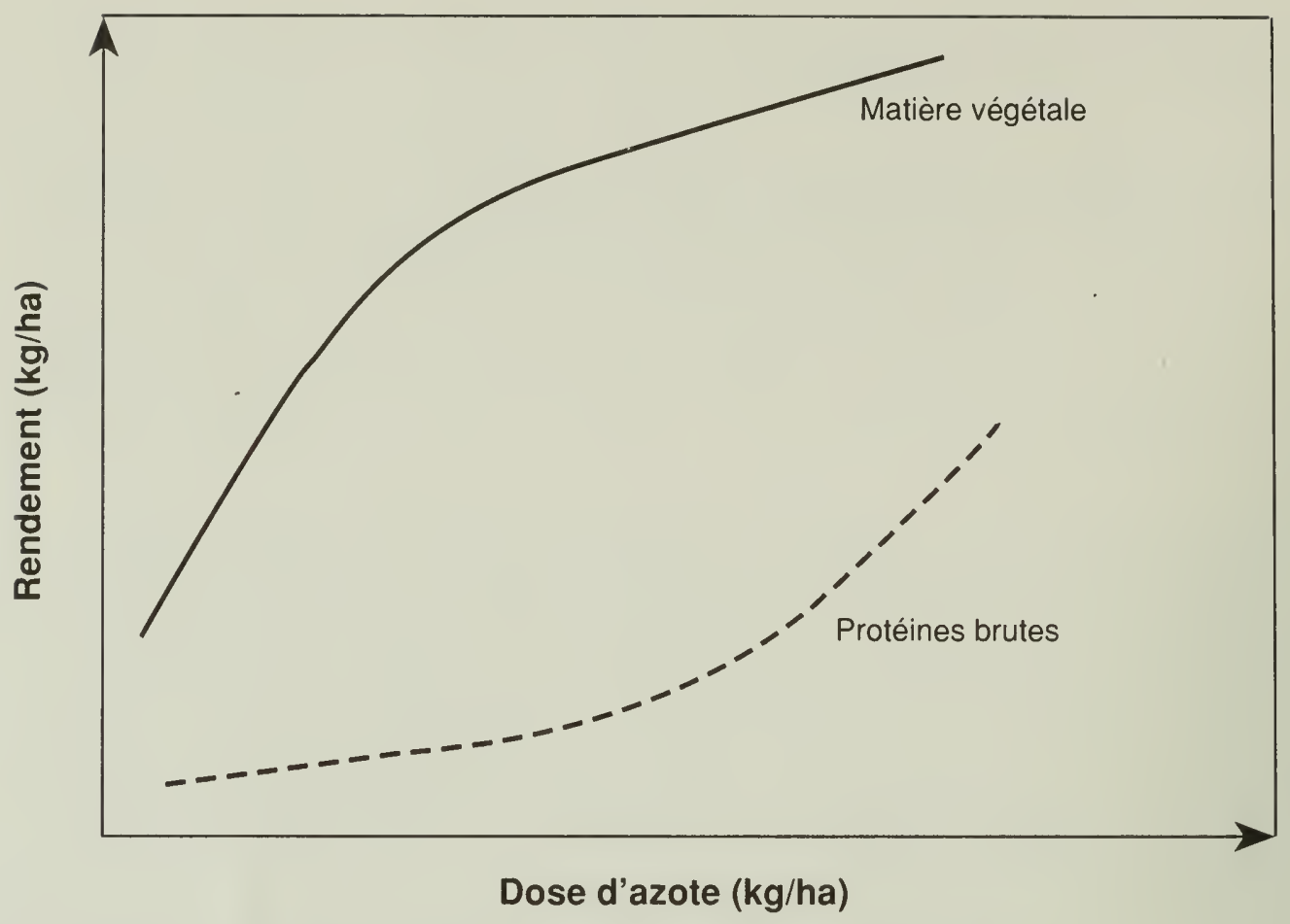

Fig. 3 Réaction générale d'une culture aux fumures azotées.

Encadré 3 De l'azote il en faut, mais pas trop non plus

- Quand l'apport d'azote est élevé, les nitrates s'accumulent dans le le vulpin des prés. Au cours d'une étude qui a duré 4 ans, à Prince George, les concentrations de nitrates ont augmenté en raison des doses des fumures azotées. Le vulpin des prés a stocké davantage de nitrates $\left(\mathrm{N}\right.$ des $\mathrm{NO}_{3}$ ) que l'alpiste roseau ou que la fléole des prés aux mêmes doses d'azote. Le vulpin des prés a la capacité de stocker les nitrates en concentrations toxiques lorsqu'il bénéficie d'apports élevés ou répétés d'azote. La concentration minimale sans danger de nitrates pour les bovins $(0,15 \%)$ a souvent été dépassée lorsque le vulpin a été fertilisé à des doses d'azote supérieures à $100 \mathrm{~kg} / \mathrm{ha}$.

- Les fortes doses d'engrais peuvent également causer d'autres problèmes. Ainsi, la verse, imputable à de fortes doses d'azote, empêche la bonne récolte des graines.

Le vulpin se révèle un choix peu judicieux dans une culture fourragère associée, en raison des problèmes de verse, tandis que les écarts entre les dates de maturité rendent difficile la conduite de la culture. 
Tableau 1 Rendement en matière sèche du vulpin des prés, à Prince George, avec apport d'azote soit au printemps seulement, soit après chaque fauche

$\mathrm{Au}$ printemps seulement

Apport d'azote Rendement
Au printemps et après chaque fauche

Apport d'azote Rendement

$(\mathrm{kg} / \mathrm{ha})$

0

45

90

180

360

\section{6}

3059

3990

5549

6794
1346

5654

6779

7109

\section{Fertilisation et rendement en matière sèche}

Le rendement du vulpin des prés, tout comme celui des autres graminées, dépend de la quantité d'éléments nutritifs assimilables dans le sol. Or, comme les graminées sont cultivées sur une large gamme de types de sols et de conditions du milieu, les besoins en fertilisants sont variables.

Parmi tous les éléments nutritifs fournis au sol, l'azote est généralement le plus en demande, le plus concentré dans la culture et le plus déterminant pour la croissance de cette dernière. La réaction générale des cultures de graminées aux apports d'azote est bien connue (fig. 3). Aux faibles doses, le rendement en matière sèche augmente de façon linéaire, tandis que la teneur en protéines brutes reste relativement constante. Aux doses élevées, le surplus d'azote augmente le rendement en protéines brutes plus que celui de la matière sèche (voir encadré 3). Dans un essai effectué à Prince George (tableau 1), les doses de 90 et de $180 \mathrm{~kg}$ d'azote à l'hectare ont permis d'augmenter le rendement en matière sèche de 300 et de $400 \%$, respectivement, dans un peuplement établi, relativement à un apport nul d'azote. Le rendement en protéines brutes a augmenté de 300 et de $500 \%$ à l'apport de 90 et de $180 \mathrm{~kg}$ d'azote à l'hectare, respectivement. On peut encore augmenter le rendement, par fumure azotée après chaque fauche. Ainsi, à Prince George, l'apport d'azote à raison de 90 et de $180 \mathrm{~kg} / \mathrm{ha}$ après chaque fauche s'est traduit par un rendement d'au moins $6800 \mathrm{~kg} / \mathrm{ha}$.

À des doses modérées (de 45 à $90 \mathrm{~kg} / \mathrm{ha}$ ), la fumure azotée printanière profite notamment à la première fauche, parce que la plus grande partie de l'engrais est assimilée facilement par la culture en 
croissance. Si les doses d'azote sont élevées ( $180 \mathrm{~kg} / \mathrm{ha}$ ) ou si les applications sont fractionnées, l'azote profite également à la seconde ou à la dernière fauche. Comme le vulpin des prés est une graminée à croissance précoce, qui peut habituellement être fauchée plus souvent dans la saison que l'alpiste roseau ou la fléole, les applications fractionnées lui semblent plus profitables. Toutefois, plus tard dans la saison, comme les jours raccourcissent et que le sol s'assèche, la croissance du vulpin réagit nettement moins aux engrais.

Un peuplement bien établi et vigoureux de vulpin des prés, dont le rendement annuel se situe aux environs de $5000 \mathrm{~kg} / \mathrm{ha}$, assimilera environ $125 \mathrm{~kg}$ d'azote, $40 \mathrm{~kg}$ de phosphore $\left(\mathrm{P}_{2} \mathrm{O}_{5}\right), 150 \mathrm{~kg}$ de potassium $\left(\mathrm{K}_{2} \mathrm{O}\right)$ et $15 \mathrm{~kg}$ de magnésium $(\mathrm{MgO})$. Une culture fourragère du vulpin a besoin de plus de fertilisation qu'un pâturage de cette graminée parce que le sol y subit une exportation plus grande des éléments nutritifs. Afin de compenser pour ces pertes, on remplace les éléments selon les recommandations fondées sur l'analyse des sols et on inspecte régulièrement la culture, à la recherche de signes de carences.

La quantité de fertilisant ajoutée à la culture contribue beaucoup au coût annuel des intrants; on décide de la quantité de fertilisant à utiliser à partir des données suivantes :

- recommandations découlant de l'analyse des sols locaux

- coût des fertilisants

- augmentation prévue du rendement

- augmentation de la qualité

- valeur des aliments pour le bétail

- valeur des semences.

Afin de sauvegarder la qualité des aliments pour le bétail, il est important de faire brouter et de récolter ainsi que de conserver le vulpin de façon convenable afin de protéger son investissement.

\section{Utilisation}

\section{Qualité}

Comme le montrent les analyses en laboratoire, la qualité chimique du vulpin des prés est très bonne. Les concentrations de protéines et de minéraux ont toujours été plus élevées chez cette graminée que chez les autres, dans les mêmes conditions. D'après la comparaison de sept graminées, y compris le vulpin, au cours d'une vaste expérience effectuée dans sept localités du centre de la Colombie-Britannique, le rendement du vulpin était en moyenne le plus faible. Pourtant, sa qualité chimique apparente était l'une des meilleures (tableau 2). Sa teneur en protéines brutes, en matière sèche digestible, en phosphore ainsi qu'en oligoéléments a toujours été supérieure à celle des autres graminées.

Le vulpin des prés est constamment plus riche en phosphore que les autres graminées cultivées dans les mêmes conditions pédologiques et autres. Lorsque l'on donne du vulpin des prés au bétail, il importe de 
Tableau 2 Rendement moyen et composition chimique du vulpin des prés et de la fléole, dans sept localités du centre-nord en ColombieBritannique, les deux plantes ayant été ensemencées au cours de trois années consécutives, puis récoltées pendant 3 ans, ce qui donne en tout 9 années-récoltes dans chaque localité

\begin{tabular}{lcc}
\hline & \multicolumn{2}{c}{ Espèces de graminée } \\
\cline { 2 - 3 } Paramètre & Vulpin des prés & Fléole \\
\hline Rendement en MS* (kg/ha) & 4480 & 6227 \\
MSD** (\%) & 70,6 & 61,4 \\
Rendement en MSD** (kg/ha) & 3180 & 3785 \\
Protéines brutes (PB) (\%) & 17,1 & 10,9 \\
Rendement en PB (kg/ha) & 758 & 658 \\
Phosphore (\%) & 0,34 & 0,22 \\
Calcium (\%) & 0,25 & 0,27 \\
Rapport du Ca au P & 0,74 & 1,21 \\
Potassium (\%) & 3,0 & 2,1 \\
Magnésium (\%) & 0,17 & 0,15 \\
Manganèse (ppm) & 79,2 & 46,6 \\
Cuivre (ppm) & 5,9 & 3,5 \\
Zinc (ppm) & 35,2 & 27,9 \\
\hline
\end{tabular}

* MS : matière sèche.

** MSD : matière sèche digestible.

vérifier le rapport du calcium au phosphore. La concentration de calcium chez cette graminée n'augmente pas autant que la concentration de phosphore, notamment aux premiers stades de la croissance. Lorsqu'on distribue du vulpin au bétail, il faut donner à ce dernier des suppléments calciques.

\section{Pâturage et production de foin ainsi que d'ensilage}

Le vulpin des prés est le mieux adapté au pâturage, parce que ses talles sont petites, rapprochées des unes des autres. Tôt dans la saison, la croissance peut dépasser le rythme de broutage, ce qui peut obliger à faucher l'excédent pour maintenir la plante dans un état végétatif qui se prête au broutage. Si la capacité de charge n'est pas suffisante pour compenser la croissance rapide au début de la saison, la plante fleurit et les feuilles vieillissent, ce qui se traduit par une accumulation de débris végétaux peu propices à la production animale.

La croissance rapide du vulpin des prés au début de la saison se traduit par un bon gain de poids chez le bétail et par une capacité de charge supérieure à celle de la fléole au cours du broutage du début du printemps. Les gains procurés par le vulpin ne persistent guère, et la capacité de charge diminue rapidement au moment où la production de 
la fléole augmente. Après la mi-juin, le gain de poids des animaux dépend davantage de la fléole que du vulpin. Vu que la croissance du vulpin est précoce, l'épiaison l'est également; le broutage devrait donc précéder l'épiaison et la perte consécutive de l'appétibilité de cette graminée (fig. 3).

Au cours du pâturage estival, la sécheresse peut empêcher la croissance du vulpin des prés dans les sols argileux lourds comme ceux du centre de l'intérieur de la Colombie-Britannique et des plateaux secs. D'autres graminées, plus tolérantes à la sécheresse, sont recommandées pour les pâturages exposés fréquemment à la sécheresse estivale. La repousse du vulpin au début de l'automne permet à cette graminée de tolérer un broutage intense, après que les autres pâturages ont été épuisés.

Même si le vulpin des prés peut être fauché pour servir de foin, d'autres graminées possèdent habituellement un meilleur rendement, après une seule fauche. Dans l'année, on peut faucher le vulpin trois ou quatre fois, mais les coûts supplémentaires de la récolte abaissent le rendement de chaque opération, ce qui rend cette solution impraticable. Le vulpin est habituellement prêt à récolter à la fin de mai ou au début de juin, lorsque les conditions météorologiques sont souvent imprévisibles. Des essais sur la sélection de la date de fauche ont montré la diminution marquée de la qualité après la fin de l'épiaison (fig. 4 et 5 ).

Le vulpin peut être récolté en vue de l'ensilage. Des essais ont montré que cette graminée se prêtait bien à l'ensilage et que les bovins la consommaient sans se faire prier. D'autres études ont besoin d'être effectuées pour mieux évaluer la qualité et la productivité de l'ensilage du vulpin des prés.

Le fait de retarder la récolte du vulpin fourrager jusqu'à ce que les conditions météorologiques soient favorables autorise la récolte des semences du vulpin. Le chaume peut être donné aux animaux comme fourrage. On résout ainsi le problème que posent les épis peu appétissants pour les animaux (voir encadré 4). 


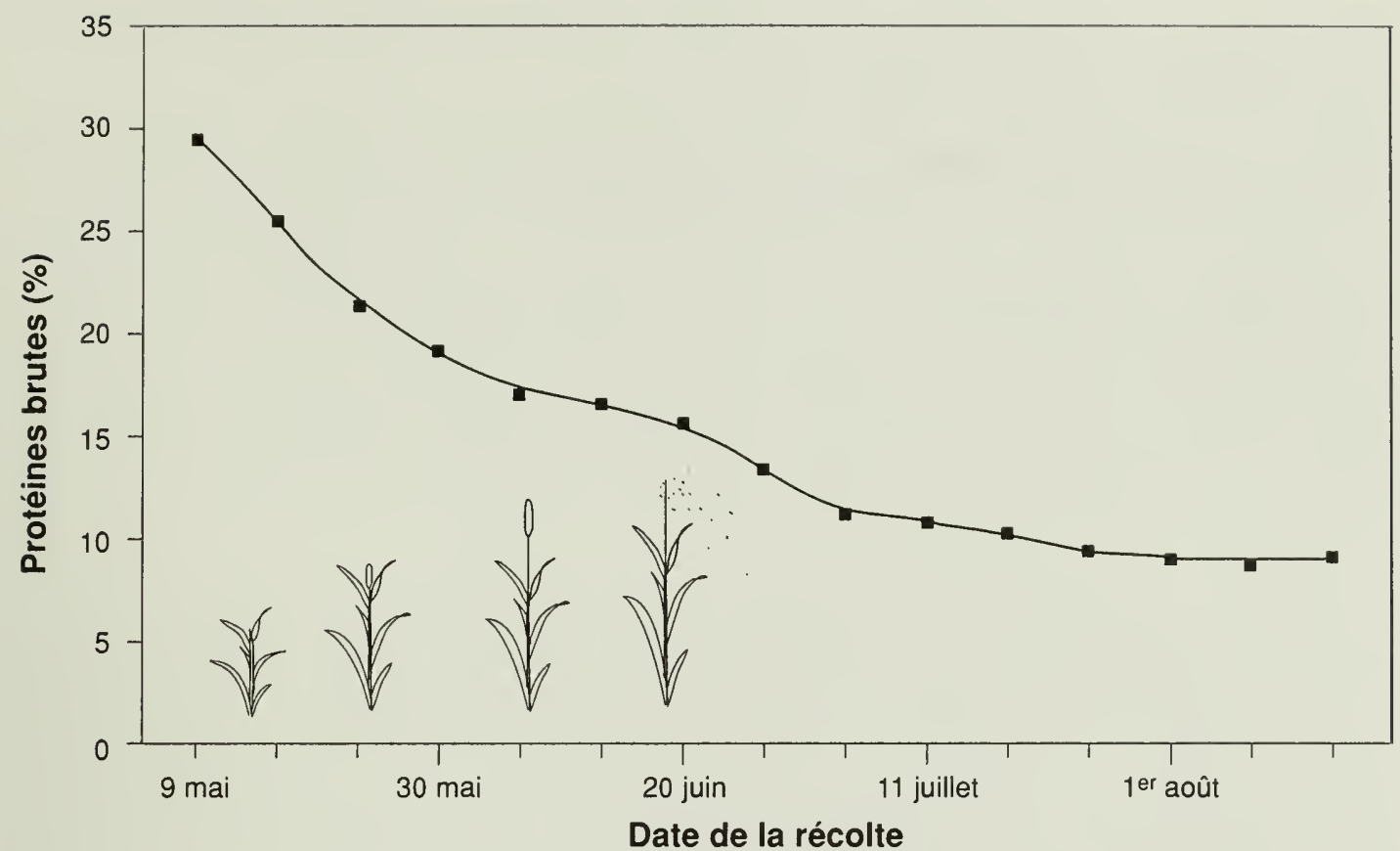

Fig. 4 Teneur en protéines brutes du vulpin des prés, au moment de la récolte, quand le champ a été fertilisé aux doses recommandées.

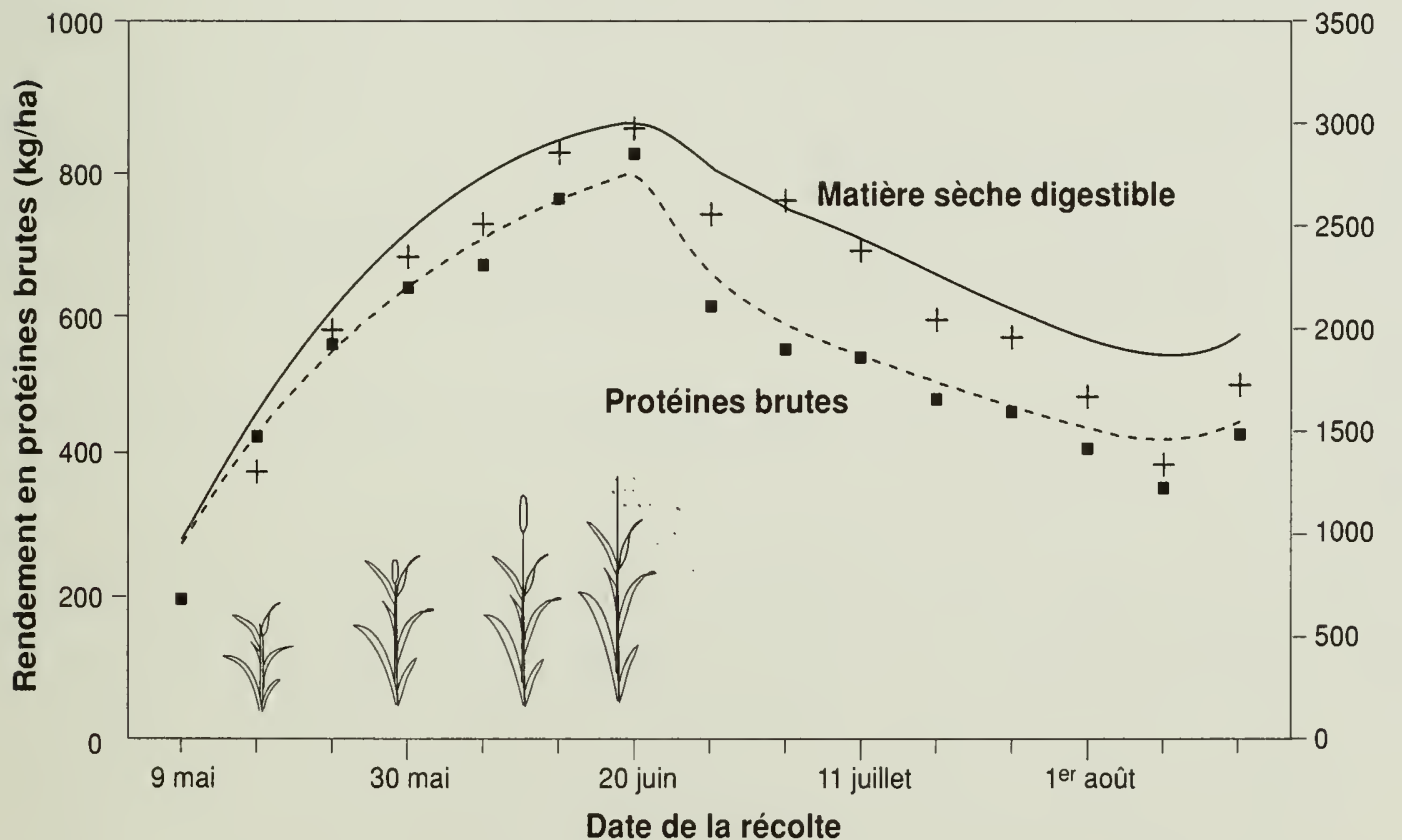

Fig. 5 Rendement en protéines brutes et en matière sèche digestible du vulpin des prés au moment de la récolte, quand le champ a été fertilisé aux doses recommandées (rendement en protéines brutes $=\%$ de protéines brutes $x$ rendement en matière sèche). 


\section{Encadré 4 Une question de goût!}

La recherche fondée sur la composition chimique montre que le vulpin des prés devrait être une excellente plante fourragère en raison de sa teneur élevée en substances nutritives, notamment en protéines. Toutefois, les producteurs du centre de l'intérieur de la ColombieBritannique ont observé que le vulpin fauché et donné à des bovins qui ont le choix đ’autres foins également est dédaigné au profit des autres graminées. Pourquoi? Nous avons pensé à mener notre petite enquête auprès des bovins et des moutons ...

- Plus cette graminée est broutée tôt, plus elle est susceptible d'être acceptée par le bétail. Dans les peuplements purs de vulpin des prés, on a observé que les moutons mangeaient de façon sélective les feuilles basilaires et rejetaient les épis et les tiges. Les bovins ne peuvent sélectionner aussi rapidement que les moutons. D'autres essais sur l'appétibilité de la plante pour les bovins et les moutons ont montré que ces animaux rejetaient généralement cette graminée dès qu'elle avait fleuri. Il existerait donc un facteur anti-qualité, non encore identifié, qui peut diminuer l'appétibilité de la graminée. Souvent le temps inclément ne permet pas la fauche avant la période recommandée, notamment dans le centre de l'intérieur de la Colombie-Britannique. Comme le chaume du vulpin passe pour être très recherché par les animaux, le facteur anti-qualité peut se trouver concentré dans l'épi. Si les champs ne peuvent pas être broutés ni fauchés avant la floraison, alors les semences devraient être récoltées directement lorsqu'elles sont à maturité et la paille pourrait être pressée pour servir de fourrage.

....Qu'en est-il des chevaux?

- Les chevaux ne trouvent pas le vulpin aussi appétissant que les autres graminées y compris la fléole et le dactyle pelotonné, si l'on se fie à des essais effectués en Grande-Bretagne.

Lorsque le vulpin est brouté dans des peuplements mélangés, il peut être rejeté. Pourquoi?

- Habituellement, on met les animaux à brouter dans des peuplements mélangés quand toutes les plantes fourragères ont atteint un bon stade de croissance. Ce peut-être alors trop tard pour le vulpin des prés! Les autres plantes fourragères peuvent être à un stade plus appétissant de la croissance. Pour surmonter le problème, il suffit de cultiver le vulpin des prés en peuplement pur et d'y faire brouter les animaux le plus tôt possible. Il peut ne pas sembler pratique de mettre le bétail dans des champs dont le sol n'est pas suffisamment ferme pour tolérer un piétinement indispensable. Toutefois la régénération de cette graminée est bonne. 


\section{Production semencière}

La production semencière du vulpin des prés se limite principalement à l'Oregon, même si on s'y adonne un peu en Alberta. Si la culture du vulpin est facile, il n'en va pas toujours de même pour la récolte et le nettoyage des semences.

Les semences duveteuses font qu'il en faut à peine plus d'un million pour un kilogramme; avec une masse volumique de $193 \mathrm{~kg} / \mathrm{m}^{3}$, le poids du boisseau est petit. Le rendement varie fortement, de 50 à $400 \mathrm{~kg} / \mathrm{ha}$, du fait des conditions météorologiques variables au moment de la récolte et de la maturité inégale des épis.

\section{Récolte}

Parce que les semences mûres du vulpin des prés tombent facilement, le rendement semencier peut être considérablement réduit. La maturité des semences ne prend qu'une quarantaine de jours et elle est irrégulière parce que les semences mûrissent du bout de l'épi en descendant. Les trois quarts de l'épi tout au plus peuvent porter des graines mûres en même temps. Le moment propice à la récolte arrive tôt, puisque la plante arrive rapidement à maturité.

La date de maturité dépend de la zone climatique, de la température, de l'humidité, du type de sol et du régime de fertilisation. A Prince George et à Rocky Mountain House (Alb.), la récolte des semences a généralement lieu vers la fin de juin. Lorsque le sol est très humide, la floraison peut être réduite et retardée.

On peut se servir de l'andainage traditionnel et d'une moissonneusebatteuse, mais la pureté des semences ne sera que de 20 à $25 \%$. En Alberta, où on produit la plus grande partie des semences de vulpin des prés, une moissonneuse spéciale (Vertec 2250) a été imaginée pour une récolte plus efficace des semences. Le rabatteur à lattes flexibles presse les épis contre une cage métallique rotative. Les semences mûres sont arrachées de la plante, tandis que les semences non mûres restent fixées à la plante non coupée. Les semences sont ensuite aspirées dans une gaine par commande pneumatique et recueillie dans une trémie. Elles sont si légères qu'il ne faut pas beaucoup d'air pour les transporter. On obtient ainsi des semences très propres, et on possède ainsi la souplesse voulue pour effectuer la récolte des semences peu après la pluie. En outre on peut laisser le chaume sur pied dans le champ. Les pertes causées par l'égrenage, l'altération du grain sur pied et les semences immatures sont considérablement réduites, et le même champ peut être récolté plus d'une fois, après que les semences résiduelles ont fini de mûrir.

\section{Manutention des semences}

Que l'on recoure à l'andainage suivi du moissonnage-battage ou simplement au moissonnage-battage, il faut sécher les semences si ces dernières sont trop mouillées. Le moissonnage-battage direct est plus 
susceptible de donner des semences humides parce que la plante est encore en pleine croissance au moment de la récolte. Des trémies d'aération pourvues de ventilateurs qui soufflent l'air au travers des semences peuvent servir au séchage des semences.

\section{Après le moissonnage-battage}

Le reste de la plante peut ensuite être coupé, comme foin, puisque sa teneur en protéines se situerait encore entre 7 et $10 \%$ si la fauche a lieu à la floraison. À Prince George, la grenaison a lieu autour du 19 juin. Si l'humidité du sol est convenable, il reste suffisamment de temps pour une bonne repousse du peuplement, qui peut alors servir de pâturage dans l'arrière-saison. Le broutage automnal ne nuit pas à la production de semences de l'année suivante.

\section{Autres utilisations}

Avec sa capacité de tolérer la submersion et de croître en terrain humide, le vulpin des prés est naturellement désigné pour les zones de nidification des anatidés, famille d'oiseaux palmipèdes. En général les canards préfèrent les herbes plus hautes que le vulpin des prés. Toutefois, parce que les anatidés préfêrent un mélange de graminées, le vulpin des prés peut être utile dans un mélange, en raison de sa croissance précoce. Le principal inconvénient est qu'il domine les autres graminées qui sont moins tolérantes à l'eau. Il se répand facilement le long des cours d'eau et peut devenir nuisible. L'ensemencement exige donc une planification soignée. Dans les terres humides où le système racinaire développé de l'alpiste roseau colmate les drains ou ralentit l'écoulement de l'eau, le vulpin des prés peut se révéler une bonne solution de rechange.

Les animaux tels que le cerf et le wapiti trouvent le vulpin des prés appétissant. Dans les régions montagnardes, ce dernier est l'une des premières graminées à croître au printemps, ce qui procure à la faune du fourrage peu après la fonte. L'espèce est bien adaptée aux pelouses alpines humides.

Pour la conservation des sols, le vulpin des prés peut être utile dans les terrains gorgés d'eau. Toutefois, d'autres graminées telles que l'alpiste roseau possèdent un meilleur système racinaire pour la stabilisation des berges.

Pour la végétalisation des lieux d'enfouissement, le vulpin des prés n'a pas donné satisfaction. La salinité élevée de beaucoup de ces lieux ralentit la croissance et le développement de la plante. 


\section{Recommandations pour l'emploi du vulpin des prés}

Le vulpin des prés n'est pas supérieur à la fléole des prés ni au dactyle pelotonné lorsque ces graminées sont bien adaptées, mais il est particulièrement utile sur les terrains trop humides pour la plupart des graminées.

- Ne le semer que dans les terrains plus humides que les autres.

- Ne pas ensemencer de grandes superficies avec cette graminée qui a besoin d'une régie attentive et de qualité.

- Mettre à brouter tôt (des suppléments calciques peuvent être nécessaires).

- Le faucher tôt pour l'utiliser en tant que foin ou ensilage, plus tôt que la plupart des autres graminées.

- À défaut de faucher le vulpin tôt, pour l'utiliser comme foin, récolter les semences avant la fauche.

\section{Remerciements}

Nous savons particulièrement gré au personnel de la Ferme expérimentale de Prince George qui, au cours des ans, a évalué le vulpin des prés dans le champ et en tant qu'aliment pour les animaux. C'est une partie de ce travail qui se trouve reproduite dans le présent guide de production. Le $\mathrm{D}^{\mathrm{r}}$ A.R. McElroy du Centre de recherches phytotechniques d'Agriculture Canada nous a fourni des données sur cette graminée cultivée en Ontario. Les $\mathrm{D}^{\mathrm{rs}}$ J.A. Robertson, D.G. Stout, A.L. van Ryswyk et D.A. Quinton de la Station de recherches de Kamloops nous ont donné des conseils utiles pour améliorer notre manuscrit. $\mathrm{M}^{\mathrm{me}}$ Ann Robertson a effectué des corrections et proposé des changements aux dernières ébauches. Le $\mathrm{D}^{\mathrm{r}}$ Walter Carlson, éleveur local, nous a transmis une partie de son expérience pratique de la récolte du foin de vulpin et de l'alimentation du bétail avec ce foin. M. Bill Smith de la Direction générale de la production et de l'inspection des aliments d'Agriculture Canada à Kelowna (C.-B.) nous a transmis des renseignements sur les herbicides. 


\section{Pour en savoir plus}

Broersma, Klaas. 1990. Meadow foxtail. Ministère de l'Agriculture et des Pêches de la Colombie-Britannique. Fiche d'information 90-22.

Broersma, Klaas; Tingle N.N. 1987. Yield and quality of seven grass species at seven locations in the central interior of British Columbia. Direction générale de la recherche, Agric. Can. Bull. Tech. 1987-14E. 63 pp.

Kline, Patricia; Broersma, Klaas. 1983. The yield, nitrogen, and nitrate content of reed canarygrass, meadow foxtail, and timothy fertilized with nigrogen. Can. J. Plant Sci. 63:943-950.

Lopetinsky, K.; Cole, D.; Okada, M.; Darivent, L. 1989. Meadow and creeping foxtail seed production in Alberta. Alberta Agriculture, Agdex 127/15-3.

Pembina Forage Association. 1982. Meadow foxtail seed harvester. Hayshaker 6(1):1-2.

Pringle, W.L.; Kline, P.M. 1982. Effects of date of cutting on yield and quality of meadow foxtail. Can. J. Plant Sci. 63:347. (résumé)

Rode, L.M. 1986. Inhibitory effect of meadow foxtail on the growth of steers. Can. J. Anim. Sci. 66:303-305.

Rode, L.M.; Pringle, W.L. 1986. Growth, digestibility and voluntary intake by yearling steers grazing timothy or meadow foxtail. Can. J. Anim. Sci. 66:463-472.

Tingle, J.N.; van Adrichem, M.C.J. 1974. Meadow foxtail lengthens the spring pasture season. Can. Agric. 19(2):26.

van Adrichem, M.C.J. 1974. Performance of meadow foxtail. Forage Notes 19(2):50-52.

Walton, Peter D. 1983. Production and management of cultivated forages. Publishing Company, Inc., Reston, Va. 


\section{TABLE DE CONVERSIONS}

Pour convertir une unité métrique, il faut multiplier le nombre par le facteur de conversion. Pour convertir une unité métrique en unité impériale, il faut diviser le nombre par le facteur de conversion.

Unités impériales

\section{Longueur}

pouce

pied

verge

mille

\section{Surface}

pouce carré $\left(\mathrm{po}^{2}\right)$

pied carré $\left(\mathrm{pi}^{2}\right)$

verge carrée

mille carré

acre

\section{Volume}

pouce cube

pied cube

verge cube

once liquide

chopine

pinte

gallon (R.-U.)

gallon (E. -U.)

\section{Masse}

$\begin{array}{ll}\text { Once } & 28 \\ \text { livre } & 0,45\end{array}$

tonne courte $(2000 \mathrm{lb})$

\section{Température}

degrés Fahrenheit

\section{Pression}

livres par pouce carré

Puissance

cheval-vapeur

\section{Vitesse}

pieds par seconde milles par heure (mph)

\section{Agriculture}

gallons par acre
pintes par acre
chopines par acre
onces liquides par acre
tonnes par acre
livres par acre
onces par acre
plants par acre

25
30
0,9
1,6

3,8

\section{Facteurs} de conversion

0,836

0,40 kilomètres par heure

1,6 kilomètres par heure $(\mathrm{km} / \mathrm{h})$

$\begin{array}{lrl}11,23 & \text { litres par hectare } & (\mathrm{L} / \mathrm{ha}) \\ 2,8 & \text { litres par hectare } & (\mathrm{L} / \mathrm{ha}) \\ 1,4 & \text { litres par hectare } & (\mathrm{L} / \mathrm{ha}) \\ 70 & \text { millilitres par hectare } & (\mathrm{mL} / \mathrm{ha}) \\ 2,24 & \text { tonnes par hectare } & (\mathrm{t} / \mathrm{ha}) \\ 1,12 & \text { kilograms par hectare } & (\mathrm{kg} / \mathrm{ha}) \\ 70 & \text { grammes par hectare } & (\mathrm{g} / \mathrm{ha}) \\ 2,47 & \text { plants par hectare } & \end{array}$




Imprimé sur du papier recyclé

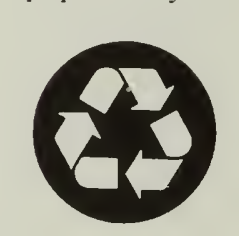

